\title{
BMJ Open Influence of organisational attributes on registered nurse contributions to well- child care: a scoping review protocol
}

\author{
Suzanne Braithwaite (D) , ${ }^{1,2}$ Julia Lukewich, ${ }^{1,3}$ Danielle Macdonald, ${ }^{1}$ Joan Tranmer $^{1}$
}

To cite: Braithwaite S, Lukewich J, Macdonald D, et al. Influence of organisational attributes on registered nurse contributions to well-child care: a scoping review protocol. BMJ Open 2021;11:e052634. doi:10.1136/ bmjopen-2021-052634

- Prepublication history and additional supplemental material for this paper are available online. To view these files, please visit the journal online (http://dx.doi.org/10.1136/ bmjopen-2021-052634).

Received 20 April 2021 Accepted 17 August 2021

Check for updates

(C) Author(s) (or their employer(s)) 2021. Re-use permitted under CC BY-NC. No commercial re-use. See rights and permissions. Published by BMJ.

${ }^{1}$ School of Nursing, Queen's University, Kingston, Ontario, Canada

${ }^{2}$ School of Health, Human, and Justice Studies, Loyalist College, Belleville, Ontario, Canada

${ }^{3}$ School of Nursing, Memorial University of Newfoundland, Saint John's, Newfoundland, Canada

Correspondence to Suzanne Braithwaite; 12sl40@queensu.ca

\section{ABSTRACT}

Introduction Universal access to preventative healthcare is essential to children's health. Registered nurses (RN) are well positioned to deliver well-child care within primary care settings; however, RN role implementation varies widely in this sector and the scope of literature that examines the influence of organisational attributes on nursing contributions to well-child care is not well understood. The aim of this scoping review is to identify the scope and characteristics of the literature related to organisational attributes that act as barriers to, or facilitators for RN delivery of well-child care within the context of primary care in high-income countries. Methods and analysis The Joanna Briggs Institute scoping review methodology will be used to conduct this review. Databases that will be accessed include Cumulative Index to Nursing and Allied Health Literature (CINAHL), MEDLINE and Embase. Inclusion criteria includes articles with a focus on RNs who deliver well-child care in primary care settings. Literature that meets this inclusion criteria will be included in the study. Covidence software platform will be used to review citations and full-text articles. Titles, abstracts and full-text articles will be reviewed independently by two reviewers. Any disagreements that arise between the reviewers will be resolved through discussion, or with an additional reviewer. Data will be extracted and organised according to the dimensions outlined in the nursing care organisation conceptual framework (NCOF). Principles of the 'best fit' framework synthesis will guide the data analysis approach and the NCOF will act as the framework for data coding and analysis.

Ethics and dissemination This scoping review will undertake a secondary analysis of data already published and does not require ethical approval. Findings will be disseminated via peer-reviewed publications and conference presentations targeting stakeholders involved in nursing practice and the delivery of well-child care. Trial registration details Braithwaite, S., Tranmer, J., Lukewich, J., \& Macdonald, D. (2021, March 31). Protocol for a Scoping Review of the Influence of Organisational Attributes on Registered Nurse Contributions to Well-child Care. https://doi.org/10.17605/0SF.IO/UZYX5.

\section{INTRODUCTION}

Universal access to preventative healthcare is essential to children's health ${ }^{1}$ and RNs are well equipped to address the unique needs of

\section{Strengths and limitations of this study}

- This protocol outlines a scoping review protocol which is informed by the Joanna Briggs Institute methodological framework

- A broad range of literature will be included in the review including, peer-reviewed journal articles and unpublished grey literature such as theses and dissertations, reports and documents produced or published by government agencies, academic institutions and other groups.

- This review is limited to articles with a title and abstract in English.

- Congruent with the scoping review approach, the quality of evidence will not be assessed as part of the study selection process.

children within the context of well-child care in the primary care setting. ${ }^{2}$ Well-child care is defined as the delivery of the combined elements of care: health supervision, anticipatory guidance, growth/development monitoring and immunisation, with the goal of promoting optimal trajectory for growth and development. ${ }^{13}$ Well-child care includes identification of influences that can positively and negatively affect health outcomes. In response to an evolving health system, it is important to understand the organisational attributes of primary care practice that influence nursing role enactment as it relates to the delivery of well-child care. Organisational attributes are defined as the features, characteristics and structures of practices in which healthcare is delivered. Organisational attributes can influence nurse and patient outcomes, influencing the extent to which nurses enact the full scope of their role. Recent systematic and scoping reviews have examined the role of nurses in primary care settings; ${ }^{4-7}$ however, only one review ${ }^{6}$ has focused specifically on well-child care. Furthermore, a recent review summarised tools that measure the influence of organisational attributes on nursing contributions to chronic disease management; ${ }^{8}$ however, no review has specifically examined 


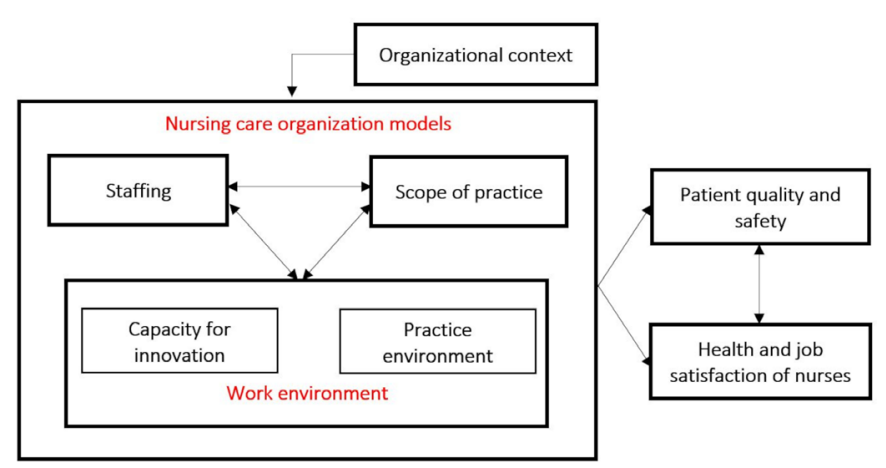

Figure 1 Nursing care Organisation framework reproduced from Dubois et al. ${ }^{9}$

organisational attributes that influence nursing contributions to well-child care.

This study will be guided by the nursing care organisation conceptual framework (NCOF) developed by Dubois and colleagues ${ }^{9}$ as presented in figure 1. A NCOF presents key organisational attributes that define a pattern of processes and allocation of resources used to deliver nursing care. The NCOF was selected for use because it integrates three distinct streams of research: nursing care administration, economics of nursing care and human resource management. The NCOF reflects the influence of three conceptual dimensions that interact as organisational correlates to influence nurse and patient outcomes. ${ }^{9}$ The three dimensions in the NCOF include: (1) staffing, (2) scope of practice and (3) work environments.

Organisational attributes that characterise nursing practice environments influence both nurse and patient outcomes. ${ }^{9}$ The concept of interest in this scoping review includes the organisational attributes that influence nursing contributions to well-child care. This includes but is not limited to attributes related to the organisational context, scope of practice and the work environment as outlined in the NCOF. ${ }^{9}$

Globally, variation exists within the literature regarding the job title of RNs employed in primary care settings. ${ }^{10}$ For the purpose of this review, the term primary care registered nurse (PCRN) will be used to classify the position. A PCRN is a registered nurse (RN) that practices in a community-based primary care setting and provides primary care services within the broader context of primary healthcare (Registered Nurses' Association of Ontario, 2012).$^{11}$ Internationally, it is unclear how many RNs are employed in primary care settings; however, RNs do engage in primary care delivery, to varying degrees, worldwide. While a portion of PCRNs hold a diploma, the $\mathrm{WHO}^{12}$ recommends a baccalaureate or bachelor's degree as the global standard for professional nursing education.

For the purpose of this review, well-child care is defined as the delivery of the following combined elements of care for children 5 years of age and younger: health supervision, anticipatory guidance, growth/development monitoring and immunisation, with the goal of promoting optimal trajectory for growth and development. ${ }^{2}$ The care includes identification of influences that can positively and negatively affect health outcomes. In Canada, the Rourke Baby Record ${ }^{13}$ is considered the best practice standard for well-child care for children 5 years of age and younger. ${ }^{14}$ Dimensions of care outlined in the Rourke Baby Record represent aspects of care that reflects delivery of well-child care. Items included in the Rourke Baby Record will be used to inform which elements of nursing care will be included in this review.

The aim of this scoping review is to identify the scope and characteristics of the literature related to organisational attributes that act as barriers to or facilitators for RN delivery of well-child care within the context of primary care in high-income countries. After a preliminary scan of the literature, it was determined that a scoping review would be the most appropriate approach to achieve this aim due to heterogeneity and amount of the available literature. A preliminary search for existing scoping reviews and systematic reviews on the topic has been conducted in CINAHL, JBI Evidence Synthesis, Cochrane Database of Systematic Reviews and Embase, yielding no systematic or scoping reviews that address the aim of this study (search conducted 9 June 2021). A scoping review is an appropriate methodology when the purpose of the review is to describe the scope and characteristics of the literature available on a specific topic. ${ }^{1516}$

\section{METHODS AND ANALYSIS}

This study will be conducted between 23 August 2021 and 23 May 2022. This study will be conducted using the approach developed by the Joanna Briggs Institute. ${ }^{17}$ This approach builds on the earlier work of Arksey and $\mathrm{O}^{\prime}$ Malley ${ }^{16}$ and Levac and colleagues. ${ }^{18}$ The approach consists of nine stages: (1) defining and aligning the research question, (2) developing and aligning the inclusion criteria, (3) describing the planned approach to evidence searching, selection, data extraction and presentation of the evidence, (4) searching the evidence, (5) selecting the evidence, (6) extracting the evidence, (7) analysis of the evidence, (8) presentation of the results and (9) summarising, making conclusions and noting implications of the findings. ${ }^{19}$ Stages one through three will be presented in this protocol. To ensure rigour in reporting the methodology, we used the Preferred Reporting Items for Systematic Reviews and Meta-analyses extension for Scoping Review (PRISMA-ScR) checklist to draft this protocol, as outlined in online supplemental file 1.

\section{Stage 1: Defining and aligning the research question}

The following research question will be addressed: What is the current state of knowledge of the organisational attributes that influence $\mathrm{RN}$ contributions to well-child care within the context of primary care in high-income countries? 
Stage 2: Developing and aligning inclusion criteria

\section{Participants}

This review will consider studies with a focus on PCRNs (general class or equivalent) who deliver well-child care in primary care settings. Additional terms will be included in the search strategy to capture internationally relevant literature. Participants in the studies may include PCRNs, their colleagues and/or key stakeholders. Data related to PCRNs with a specialty in their area of practice (eg, nurse-midwives) will be included in the review and reported independently from findings related to PCRNs without a specialty. Data specific to nurse practitioners or licensed practical nurses/registered practical nurses will be excluded from the review. Where specific nursing designation is not specified, data will be excluded.

\section{Concept}

This review will include data related to organisational attributes that influence nursing contributions to wellchild care. Based on the $\mathrm{NCOF},{ }^{9}$ this will include, but is not limited to, attributes related to the organisational context, staffing, scope of practice and the work environment. Studies with a focus on nursing, roles, activities and scope of practice will be included. Data related to any dimension of well-child care as outlined in the Rourke Baby Record will be included. Where the focus of care includes care of un-well children, including children with a terminal illness, severe psychiatric or chronic disorder, the study will be excluded from the review.

\section{Context}

Due to health service delivery differences between highincome and middle to low-income countries, this review will be restricted to primary care settings in high-income countries to isolate the context of interest. Studies conducted in countries classified as high-income economies by the World Bank ${ }^{20}$ will be included. Primary care is defined by Starfield as the sector of healthcare in which individuals first establish contact with the health system for all new needs and problems, are provided personfocused care over time for all common needs and receive coordination and integration of health services provided elsewhere by other members of the healthcare team. ${ }^{21}$ Studies conducted in settings classified as primary care according to Starfield's definition ${ }^{21}$ will be included in this review. Studies with a focus on telehealth will be included where the study setting meets the definition of primary care. Where a study includes multiple sectors, such as acute care and primary care, only data related to primary care will be included in the review. This review will exclude studies not focused on nursing within the primary care setting. Where a study includes multiple sectors, such as acute care and primary care, but does not categorise findings as such, the study will be excluded from this review.

\section{Stage 3: Describing the planned approach}

Qualitative studies, including feminist research, grounded theory, phenomenology, qualitative description, ethnography and action research, will be considered. Quantitative studies will also be considered for inclusion including randomised controlled trials, non-randomised controlled trials, analytical observational studies including prospective and retrospective cohort studies, before and after studies and interrupted time-series studies, case-control studies and analytical cross-sectional studies and descriptive observational study designs including case series, descriptive cross-sectional studies and individual case reports. Depending on the research question, systematic reviews will be considered. Text and opinion papers will also be considered for inclusion in this scoping review.

\section{Search strategy}

The three step strategy outlined in the JBI Manual for Evidence Synthesis ${ }^{19}$ will be utilised. The search strategy will aim to locate both unpublished and published studies. First, an initial limited search was undertaken in CINAHL to identify articles on the topic. The index terms used to describe the articles, and the text words contained in the titles and abstracts of relevant articles were used to develop a full search strategy for CINAHL, MEDLINE and Embase (see table 1). The search strategy will be adapted for each included database and/or information source, including all identified keywords and index terms. A second search will be undertaken across all included databases, using all identified keywords and index terms. Third, the reference list of all included sources of evidence will be screened for additional studies. To improve the relevancy of the results, titles and abstracts will be searched.

Studies with a title and abstract in English will be included in this review. It is acknowledged that this approach will result in an inherent bias towards English speaking countries and/or researchers which will be addressed in the findings. Only studies available in English will be included. To support the relevance of the review to today's health system landscape, the review will be limited to literature published within 20 years.

\section{Information sources}

The databases to be searched include CINAHL, MEDLINE and Embase. Sources of unpublished grey literature include theses and dissertations, reports, documents produced and published by government agencies, academic institutions and other groups that are not distributed or indexed by commercial publishers. ${ }^{17}$ Conference abstracts will be excluded from this review.

\section{Study/source of evidence selection}

Following the search, all identified citations will be collated and uploaded into Covidence Systematic Review software (Veritas Health Innovation, Melbourne, Australia), and duplicates removed. Prior to beginning the selection process, reviewers will engage in a pilot process. The JBI framework for pilot testing will be followed. ${ }^{17}$ First, a 


\begin{tabular}{|c|c|}
\hline Databases & Search terms \\
\hline CINAHL & $\begin{array}{l}\text { 1. Nurses }(\mathrm{SH}) \\
\text { 2. Nurs* }(\mathrm{KW}) \\
\text { 3. Registered nurse }(\mathrm{SH}) \\
\text { 4. Registered nurs }{ }^{\star}(\mathrm{KW}) \\
\text { 5. } 1 \text { or } 2 \text { or } 3 \text { or } 4 \\
\text { 6. Primary health care }(\mathrm{SH}) \\
\text { 7. Primary health care }(\mathrm{KW}) \\
\text { 8. Primary care }(\mathrm{KW}) \\
\text { 9. Family practice }(\mathrm{KW}) \\
\text { 10. General practice }(\mathrm{SH}) \\
\text { 11. General practice }(\mathrm{KW}) \\
\text { 12. } 6 \text { or } 7 \text { or } 8 \text { or } 9 \text { or } 10 \\
\text { 13. Maternal child health care }(\mathrm{SH}) \\
\text { 14. Maternal child health care }(\mathrm{KW}) \\
\text { 15. Child health services }(\mathrm{KW}) \\
\text { 16. Child health care }(\mathrm{SH}) \\
\text { 17. Child health care }(\mathrm{KW}) \\
\text { 18. Infant care }(\mathrm{SH}) \\
\text { 19. Infant care }(\mathrm{KW}) \\
\text { 20. Well-child care }(\mathrm{KW}) \\
\text { 21. Well-baby care }(\mathrm{KW}) \\
\text { 22. Rourke }(\mathrm{KW}) \\
\text { 23. } 12 \text { or } 13 \text { or } 14 \text { or } 15 \text { or } 16 \text { or } 17 \text { or } 18 \text { or } 19 \text { or } 20 \text { or } \\
21 \text { or } 22 \\
\text { 24. } 5 \text { and } 12 \text { and } 23\end{array}$ \\
\hline Embase & $\begin{array}{l}\text { 1. Nurses }(\mathrm{SH}) \\
\text { 2. Nurs* }(\mathrm{KW}) \\
\text { 3. Registered nurs* }(\mathrm{KW}) \\
\text { 4. } 1 \text { or } 2 \text { or } 3 \\
\text { 5. Primary health care }(\mathrm{SH}) \\
\text { 6. Primary health care }(\mathrm{KW}) \\
\text { 7. Primary care }(\mathrm{KW}) \\
\text { 8. Family practice }(\mathrm{SH}) \\
\text { 9. Family practice }(\mathrm{KW}) \\
\text { 10. General practice }(\mathrm{KW}) \\
\text { 11. } 5 \text { or } 6 \text { or } 7 \text { or } 8 \text { or } 9 \text { or } 10 \\
\text { 12. Maternal child care }(\mathrm{SH}) \\
\text { 13. Maternal child care }(\mathrm{KW}) \\
\text { 14. Child health services }(\mathrm{KW}) \\
\text { 15. Child health care }(\mathrm{KW}) \\
\text { 16. Infant care }(\mathrm{SH}) \\
\text { 17. Infant care }(\mathrm{KW}) \\
\text { 18. Well-child care }(\mathrm{KW}) \\
\text { 19. Well-baby care }(\mathrm{KW}) \\
\text { 20. Rourke (KW) } \\
\text { 21. } 12 \text { or } 13 \text { or } 14 \text { or } 15 \text { or } 16 \text { or } 17 \text { or } 18 \text { or } 19 \text { or } 20 \\
\text { 22. } 4 \text { and } 11 \text { and } 21\end{array}$ \\
\hline
\end{tabular}

MEDLINE $\begin{aligned} & \text { 1. Nurses }(\mathrm{SH}) \\ & \text { 2. Nurs }(\mathrm{KW}) \\ & \text { 3. Registered nurs }{ }^{*}(\mathrm{KW}) \\ & \text { 4. } 1 \text { or } 2 \text { or } 3 \\ & \text { 5. Primary health care }(\mathrm{KW}) \\ & \text { 6. Primary care }(\mathrm{KW}) \\ & \text { 7. Family practice }(\mathrm{SH}) \\ & \text { 8. Family practice }(\mathrm{KW}) \\ & \text { 9. General practice }(\mathrm{SH}) \\ & \text { 10. General practice }(\mathrm{KW}) \\ & \text { 11. } 5 \text { or } 6 \text { or } 7 \text { or } 8 \text { or } 9 \text { or } 10 \\ & \text { 12. Maternal child health care }(\mathrm{KW}) \\ & \text { 13. Child health services }(\mathrm{SH}) \\ & \text { 14. Child health services }(\mathrm{KW}) \\ & \text { 15. Child health care }(\mathrm{KW}) \\ & \text { 16. Infant care }(\mathrm{SH}) \\ & \text { 17. Infant care }(\mathrm{KW}) \\ & \text { 18. Well-child care }(\mathrm{KW}) \\ & \text { 19. Well-baby care }(\mathrm{KW}) \\ & \text { 20. Rourke (KW) } \\ & \text { 21. } 12 \text { or } 13 \text { or } 14 \text { or } 15 \text { or } 16 \text { or } 17 \text { or } 18 \text { or } 19 \text { or } 20 \\ & \text { 22. } 4 \text { and } 11 \text { and } 21\end{aligned}$

$\mathrm{KW}$, keyword; SH, subject heading. random sample of 25 articles/abstracts will be selected. Each reviewer will independently screen these according to the protocol outlined above. Once the screening is complete, the reviewers will meet to discuss discrepancies and make modifications to the protocol inclusion/exclusion criteria as required. Once $75 \%$ or greater agreement is achieved, the reviewers will begin the screening process.

Following the pilot test, two or more independent reviewers will screen titles and abstracts for assessment against the inclusion criteria for the review. Sources identified as potentially relevant will be retrieved in full. Their citation details will be imported into the JBI System for the Unified Management, Assessment and Review of Information (JBI SUMARI). ${ }^{17}$ Two independent reviewers will assess the full text of selected citations. Citations will be assessed in detail against the inclusion criteria. The reasons for exclusion of citations that do not meet the inclusion criteria on review of the full text will be recorded and reported in the scoping review. At each stage of the selection process, disagreements that arise between the reviewers will be resolved through discussion, or with an additional reviewer. The results of the search and the study inclusion process will be presented in a PRISMA-ScR flow diagram ${ }^{22}$ and reported in full in the scoping review.

\section{Data extraction}

The NCOF framework will inform data extraction and synthesis approaches. Data will be extracted from papers included in the scoping review by two or more independent reviewers using a data extraction tool developed by the reviewers. The data extracted will include specific details about the participants, concept, context, study methods and key findings relevant to the review questions. A data extraction table template has been created to chart the details of the studies and key findings of interest (see table 2). Development of the data extraction table was influenced by the JBI data extraction instrument template for scoping reviews. ${ }^{19}$ Organisation of data extraction is informed by the NCOF, the conceptual

\section{Table 2 Data extraction table template}

\section{Source details}

Study citation

Country

Context

Document type

Design

Participants (age, sex, number)

\section{Results}

Delivery of well-child care (scope of practice)

Practice environment

Capacity for innovation

Staffing 
framework informing this study. A preliminary data extraction pilot test will be completed with three sources to ensure consistency between reviewers and agreement in interpretation of the findings. At this stage, the data extraction table may be revised if necessary.

In this study, we are interested in descriptive data; therefore, our focus in this pilot will be on ensuring proper interpretation of source data and appropriate utilisation of the extraction template. The draft data extraction tool will be modified and revised as necessary during the process of extracting data from each included evidence source. Modifications will be detailed in the final scoping review report. Any disagreements that arise between the reviewers will be resolved through discussion, or with an additional reviewer. If appropriate, authors of papers will be contacted to request missing or additional data, where required.

\section{Data analysis and presentation}

Consistent with scoping review methodology, data will be descriptively mapped rather than thematically synthesised. Analysis will characterise the quantity and quality of the literature. ${ }^{23}$ Summary of included literature will be presented in a table including the following information: purpose, participants, setting, type of study and key findings. The 'best fit' framework synthesis outlined by Carroll and colleagues ${ }^{24}$ is a pragmatic, matrix-based method for data analysis involving the organisation of data according to an a priori framework. ${ }^{24}{ }^{25}$ This approach to data analysis provides the opportunity to map findings according to the a priori dimensions outlined in the NCOF. ${ }^{9}$ Principles of the 'best fit' framework ${ }^{24}$ will guide the data analysis approach and the NCOF will act as the a priori framework used for data analysis.

\section{Limitations}

This review is limited to articles with a title and abstract in English. It is also limited to the socioeconomic landscape of high-income earning countries. We also acknowledge that variability in $\mathrm{RN}$ credentialing exists across countries, proving it difficult to generalise findings. Finally, congruent with scoping review methodology, the quality of the sources will not be assessed as part of the study selection process.

\section{Nursing practice and policy implications}

Primary care is considered a key characteristic of a high performing health system, and access to preventative healthcare for children is associated with improved health and system outcomes. Appropriate enactment of the RN role is needed to address the health needs of children in the primary care sector. This scoping review has potential to uncover the scope and characteristics of the literature related to the organisational attributes that influence RN contributions to well-child care. Findings from this study have the potential to influence policy decisions regarding organisational attributes that influence nursing role enactment. Gaps identified in this scoping review may further influence future research in this area of study.

\section{Patient and public involvement}

Patients and the public were not involved in the design of this protocol. Once the scoping review has been complete, the public will be involved in the dissemination plan.

\section{ETHICS AND DISSEMINATION}

This scoping review will undertake a secondary analysis of data already published and does not require ethical approval. Findings will be disseminated via peer-reviewed publication and conference presentation targeting stakeholders involved in nursing practice and delivery of wellchild care.

Contributors SB conceived the idea and was responsible for the initial design of this study. SB, JT, JL and DM contributed to the study design, development of the research question and development of the protocol. SB led the writing of the protocol under the supervision of JT. SB, JT, JL and DM contributed to and approved the final version of this protocol.

Funding The authors have not declared a specific grant for this research from any funding agency in the public, commercial or not-for-profit sectors.

Competing interests None declared.

Patient consent for publication Not required.

Provenance and peer review Not commissioned; externally peer reviewed.

Supplemental material This content has been supplied by the author(s). It has not been vetted by BMJ Publishing Group Limited (BMJ) and may not have been peer-reviewed. Any opinions or recommendations discussed are solely those of the author(s) and are not endorsed by BMJ. BMJ disclaims all liability and responsibility arising from any reliance placed on the content. Where the content includes any translated material, BMJ does not warrant the accuracy and reliability of the translations (including but not limited to local regulations, clinical guidelines, terminology, drug names and drug dosages), and is not responsible for any error and/or omissions arising from translation and adaptation or otherwise.

Open access This is an open access article distributed in accordance with the Creative Commons Attribution Non Commercial (CC BY-NC 4.0) license, which permits others to distribute, remix, adapt, build upon this work non-commercially, and license their derivative works on different terms, provided the original work is properly cited, appropriate credit is given, any changes made indicated, and the use is non-commercial. See: http://creativecommons.org/licenses/by-nc/4.0/.

\section{ORCID iD}

Suzanne Braithwaite http://orcid.org/0000-0002-6801-5036

\section{REFERENCES}

1 Guttmann A, Shipman SA, Lam K, et al. Primary Care Physician Supply and Children's Health Care Use, Access, and Outcomes: Findings From Canada. Pediatrics 2010;125:1119-26.

2 Warmels G, Johnston S, Turley J. Improving team-based care for children: shared well child care involving family practice nurses. Prim Health Care Res Dev 2017;18:507-14.

3 Guttmann Aet al. Primary care for children. In: Primary care in Ontario. Toronto, ON: Institute for Clinical Evaluative Sciences, 2006: 35-52.

4 Curnew DR, Lukewich J. Nursing within primary care settings in Atlantic Canada: a scoping review. Sage Open 2018;8:215824401877437.

5 Martin-Misener R, Valaitis R, Wong ST, et al. A scoping literature review of collaboration between primary care and public health. Prim Health Care Res Dev 2012;13:327-46.

6 Turley J, Vanek J, Johnston S, et al. Nursing role in well-child care: systematic review of the literature. Can Fam Physician 2018;64:e169-80. 
7 Norful A, Martsolf G, de Jacq K, et al. Utilization of registered nurses in primary care teams: a systematic review. Int J Nurs Stud 2017;74:15-23.

8 Lukewich J, Corbin R, VanDenKerkhof EG, et al. Identification, summary and comparison of tools used to measure organizational attributes associated with chronic disease management within primary care settings. J Eval Clin Pract 2014;20:1072-85.

9 Dubois C-A, D'Amour D, Tchouaket E, et al. A taxonomy of nursing care organization models in hospitals. BMC Health Serv Res 2012;12:286.

10 Barrett Cet al. Job titles and education requirements of registered nurses in primary care: an international document review. Int J Nur Stud 2021

11 Registered Nurses' Association of Ontario. Primary solutions for primary care: maximizing and expanding the role of the primary care nurse in Ontario. Toronto. Ontario: Registered Nursing Association, 2012.

12 World Health Organization. Global standards for the initial education of professional nurses and midwives, 2009. Available: https://www. who.int/hrh/resources/standards/en/

13 Rourke SL, Leduc D, Rourke J. Rourke baby record: evidence-based infant/child health maintenance., 2020. Available: https://www. rourkebabyrecord.ca/pdf/RBR\%202020\%20NAT-EN-3vpp-BLK-Apr29.pdf

14 Kuo AA, Inkelas M, Lotstein DS, et al. Rethinking well-child care in the United States: an international comparison. Pediatrics 2006;118:1692-702.
15 Munn Z, Peters MDJ, Stern C, et al. Systematic review or scoping review? Guidance for authors when choosing between a systematic or scoping review approach. BMC Med Res Methodol 2018;18:143-7.

16 Arksey H, O'Malley L. Scoping studies: towards a methodological framework. Int J Soc Res Methodol 2005;8:19-32.

17 Anromataris E, Munn Z. JBI manual for evidence synthesis. JBI, 2020.

18 Levac D, Colquhoun H, O'Brien KK. Scoping studies: advancing the methodology. Implement Sci 2010;5:69.

19 Peters M. Chapter 11: Scoping review. In: Aromataris E, Munn Z, eds. JBI manual for evidence synthesis, 2020.

20 The World Bank. World bank country and lending groups., 2020. Available: https://datahelpdesk.worldbank.org/knowledgebase/ articles/906519-world-bank-country-and-lending-groups

21 Starfield B. Primary care: balancing health needs, services, and technology. Rev. ed. New York: Oxford University Press, 1998.

22 Tricco AC, Lillie E, Zarin W, et al. PRISMA extension for scoping reviews (PRISMA-ScR): checklist and explanation. Ann Intern Med 2018;169:467-73.

23 Grant MJ, Booth A. A typology of reviews: an analysis of 14 review types and associated methodologies. Health Info Libr J 2009;26:91-108.

24 Carroll C, Booth A, Leaviss J, et al. "Best fit" framework synthesis: refining the method. BMC Med Res Methodol 2013;13:37.

25 Dixon-Woods M. Using framework-based synthesis for conducting reviews of qualitative studies. BMC Med 2011;9:39. 